\title{
Measurement and control of emergent phenomena emulated by resistive- capacitive networks, using fractionalorder internal model control and external adaptive control
}

Article

Published Version

Galvão, R. K. H. and Hadjiloucas, S. (2019) Measurement and control of emergent phenomena emulated by resistivecapacitive networks, using fractionalorder internal model control and external adaptive control. Review of Scientific Instruments, 90. 103003. ISSN 0034-6748 doi: https://doi.org/10.1063/1.5097743 Available at https://centaur.reading.ac.uk/86868/

It is advisable to refer to the publisher's version if you intend to cite from the work. See Guidance on citing.

To link to this article DOI: http://dx.doi.org/10.1063/1.5097743

Publisher: AIP

All outputs in CentAUR are protected by Intellectual Property Rights law, including copyright law. Copyright and IPR is retained by the creators or other copyright holders. Terms and conditions for use of this material are defined in the End User Agreement. 


\section{www.reading.ac.uk/centaur}

\section{CentAUR}

Central Archive at the University of Reading

Reading's research outputs online 


\section{Measurement and control of emergent phenomena emulated by resistive- capacitive networks, using fractional- order internal model control and external adaptive control}

Cite as: Rev. Sci. Instrum. 90, 103003 (2019); https://doi.org/10.1063/1.5097743

Submitted: 28 March 2019 . Accepted: 23 July 2019 . Published Online: 21 October 2019

Roberto K. H. Galvão (D), and Sillas Hadjiloucas (D)

\section{Challenge us.} What are your needs for periodic signal detection?

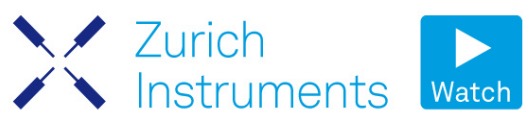

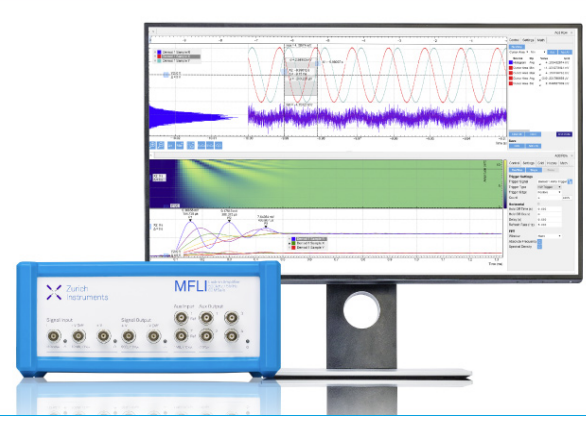

90, 103003 


\title{
Measurement and control of emergent phenomena emulated by resistive-capacitive networks, using fractional-order internal model control and external adaptive control
}

\author{
Cite as: Rev. Sci. Instrum. 90, 103003 (2019); doi: 10.1063/1.5097743 \\ Submitted: 28 March 2019 - Accepted: 23 July 2019 • \\ Published Online: 21 October 2019
}

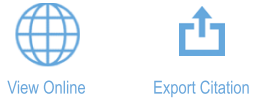

Roberto K. H. Galvão ${ }^{1, a)}$ (D) and Sillas Hadjiloucas ${ }^{2, a), b)}$ (D)

\begin{abstract}
AFFILIATIONS
'Department of Electronics Engineering, Instituto Tecnológico de Aeronáutica, São José dos Campos, SP, Brazil

${ }^{2}$ School of Biological Sciences, Department of Biomedical Engineering, The University of Reading, Reading RG6 6AY,

United Kingdom
\end{abstract}

a) Contributions: R. K. H. Galvão and S. Hadjiloucas contributed equally to this work.

b) Author to whom correspondence should be addressed: s.hadjiloucas@reading.ac.uk

\begin{abstract}
A fractional-order internal model control technique is applied to a three-dimensional resistive-capacitive network to enforce desired closedloop dynamics of first order. In order to handle model mismatch issues resulting from the random allocation of the components within the network, the control law is augmented with a model-reference adaptive strategy in an external loop. By imposing a control law on the system to obey first order dynamics, a calibrated transient response is ensured. The methodology enables feedback control of complex systems with emergent responses and is robust in the presence of measurement noise or under conditions of poor model identification. Furthermore, it is also applicable to systems that exhibit higher order fractional dynamics. Examples of feedback-controlled transduction include cantilever positioning in atomic force microscopy or the control of complex de-excitation lifetimes encountered in many types of spectroscopies, e.g., nuclear magnetic, electron-spin, microwave, multiphoton fluorescence, Förster resonance, etc. The proposed solution should also find important applications in more complex electronic, microwave, and photonic lock-in problems. Finally, there are further applications across the broader measurement science and instrumentation community when designing complex feedback systems at the system level, e.g., ensuring the adaptive control of distributed physiological processes through the use of biomedical implants.
\end{abstract}

Published under license by AIP Publishing. https://doi.org/10.1063/1.5097743

\section{INTRODUCTION}

Fractional order analysis is an emergent interdisciplinary topic with several applications when there are nonlocal interactions of physical phenomena as well as in the control of distributed systems. A typical example is the modeling of power systems ${ }^{1,2}$ for energy transmission and storage $e^{3,4}$ and, in particular, supercapacitor modeling and discharge control. ${ }^{5-11}$ Distributed system postulations are also often encountered in the modeling of biological processes. ${ }^{12-22}$ A review of fractional order circuits that may be used to emulate biological processes has been discussed in the article by Freeborn ${ }^{2}$ and the references therein. Other applications include the modeling and analysis of dielectric responses ${ }^{23-28}$ including those of memristive nonlinear circuits. ${ }^{29}$ For example, fractional order calculus naturally explains dielectric behavior of complex materials while taking into consideration long-range interacting dipoles. ${ }^{30-33}$ Furthermore, fractional order circuits are finding their way in many filtering applications, ${ }^{34,35}$ as well as in communications.

As distributed system modeling has evolved, there has also been a surge in new system identification approaches, using state variable filters ${ }^{38}$ or using continuous order distributions, ${ }^{39}$ and there are specific dedicated tool boxes, ${ }^{40,41}$ e.g., FOMCON and modulation functions available to the user. ${ }^{42}$ In order to account for emergent responses as encountered in biomedical applications ${ }^{43}$ 
and the associated more complex dynamics ${ }^{44}$ or model cyberphysical systems, ${ }^{45}$ which incorporate signals in multiple physical domains, new system identification approaches are also under development.

As discussed in Ref. 46, it is possible to emulate systems with complex dynamics using RC networks. Such circuits can be designed specifically to have tailored responses with rather complex dynamics. ${ }^{47-53}$ Furthermore, they enable analog realizations of fractionalorder controllers as discussed in the work by Petrás ${ }^{54}$ as well as the works by Podlubny et al., ${ }^{55}$ Charef, ${ }^{56}$ and Luo and Chen. ${ }^{57}$ Further advances on the stability of linear systems with fractional-order elements were extensively discussed by Petrás ${ }^{58}$ as well as Radwan et $a l^{59}$ A descriptor system approach was proposed by Tavazoei and Haeri. ${ }^{60}$ As fractional order controllers are becoming more widespread, there are also new opportunities to incorporate them in more elaborate control strategies.

Internal model control (IMC) is a particularly efficient control strategy, which has been gaining increasing popularity. ${ }^{61-65}$ Nowadays, the basic IMC formulations have been extended to incorporate a neural network to account for system nonlinearities ${ }^{66,67}$ including input saturation, ${ }^{68}$ as well as feedforward and feedback linearization strategies. ${ }^{69}$ The extension to the fractional-order case has also been addressed in the literature. ${ }^{70-73}$

The present work is concerned with the use of a fractionalorder IMC formulation for the control of a three-dimensional RC analog circuit. The motivation for performing studies with such circuits stems from the large number of their possible applications across physical sciences. For example, an interesting property of the fractal nature of semi-infinite RC ladder networks is that they can be used to model systems described by fractional order integro-differential equations. ${ }^{74-78}$ Currently, there are several physical systems whose behavior can be compactly described using such models and the observed responses can be directly associated with specific macroscopic or microscopic scale fractal geometrical structures. ${ }^{79}$ Examples include long distributed lines, ${ }^{80}$ electromechanical viscoelastic materials, ${ }^{81,82}$ or materials employed in energy storage applications. ${ }^{83,84}$ Moreover, systems subjected to processes described using statistical mechanics such as Brownian motion, $1 / f$ noise, diffusion, or chaotic oscillators ${ }^{85-89}$ also may display a fractional order behavior that can be emulated using such circuits.

In the current work, a simple yet elegant IMC design solution based on a fractional order system model of these networks is employed. In order to handle model mismatch issues resulting from the random component allocation within the RC network, we propose an augmentation of the control law with a model-reference adaptive control (MRAC) strategy in an external loop. In Secs. II and III, we discuss the basic IMC topology and the introduction of the external adaptive controller to account for the mismatch between the internal model and the actual system dynamics. A preliminary example is presented to illustrate the main features of the proposed control law by using a system model with multiple fractional exponents. Changes in the model coefficients and exponents are introduced to investigate the robustness with respect to model mismatch arising from poor identification. The 3-D RC network case study is then presented under nominal conditions, as well as in the presence of increased model mismatch and measurement noise. The added benefits of the MRAC approach over simple fractional order IMC are showcased by demonstrating a faster error convergence rate to zero. The tuning space of the proposed control law is also discussed. The formulation is generic and may be adopted to various measurement science applications.

\section{INTERNAL MODEL CONTROL TOPOLOGY}

Figure 1 depicts the internal model control (IMC) topology employed in the current study. In this block diagram, the Laplace transforms $U(s)$ and $Y(s)$ correspond to the input voltage to the $3 \mathrm{D}-\mathrm{RC}$ network and the resulting current signal, respectively. The Laplace transform of the reference signal is denoted by $R(s)$.

From the topology in Fig. 1, the transfer function $G_{C L}(s)=Y(s) / R(s)$ for the closed-loop system is given by

$$
G_{C L}(s)=\frac{G(s) Q(s)}{1+Q(s)[G(s)-\hat{G}(s)]} .
$$

If there is no model mismatch, i.e., $\hat{G}(s)=G(s)$, the expression (1) becomes simply $G_{C L}(s)=G(s) Q(s)$. Therefore, given a desired closed-loop transfer function $G_{D}(s)$, the controller can be designed by choosing

$$
Q(s)=\frac{G_{D}(s)}{\hat{G}(s)} .
$$

In this work, the internal model will be cast in the form of a fractional-order transfer function $\hat{G}(s)$, as recently proposed in the IMC literature, ${ }^{72}$ in order to better match the dynamics of the $3 \mathrm{D}$ RC network. The desired closed-loop dynamics will be specified in the form of the following first-order transfer function:

$$
G_{D}(s)=\frac{Y(s)}{R(s)}=\frac{b_{D}}{s+a_{D}}
$$

with $a_{D}=b_{D}$ so that the output converges to the setpoint with time constant $1 / a_{D} .^{90}$

It is worth noting that the transfer function $G(s)$ of the system to be controlled is not used in the design of the controller. Instead, the design is based on an estimated transfer function $\hat{G}(s)$, which can be obtained by using fractional-order system identification methods, as in Ref. 91. The transfer function $Q(s)$ is calculated through (2), given the desired closed loop transfer function $G_{D}(s)$ with the value $a_{D}=b_{D}$ chosen by the designer.

Model mismatch between $G(s)$ and $\hat{G}(s)$ could be evaluated by comparing the open-loop responses of the actual system and the

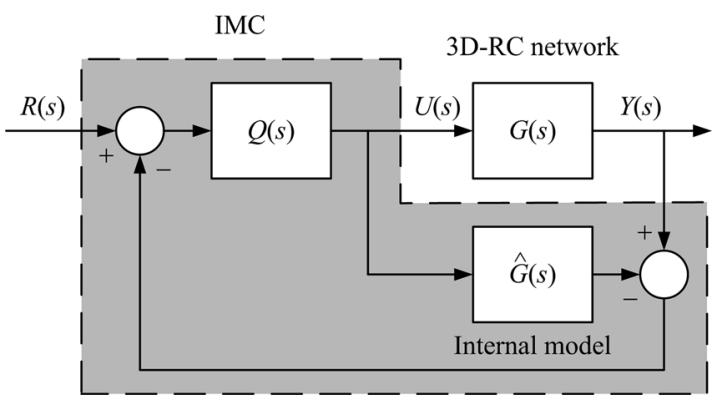

FIG. 1. Control loop topology. 
identified model, as in Ref. 91. However, the present work does not require a detailed characterization of the model mismatch. Indeed, an external adaptive control loop is proposed to compensate for such a mismatch, as will be now described.

\section{PROPOSED AUGMENTATION OF THE CONTROL LAW WITH AN EXTERNAL ADAPTIVE CONTROL LOOP}

Figure 2 presents the external model-reference adaptive control (MRAC) loop, ${ }^{92}$ which will be employed to account for model mismatch in the IMC scheme. In the present case, the IMC controller was already designed in order to follow the desired dynamics described by the reference model $G_{D}(s)$ in (6). However, due to possible mismatches between $G(s)$ and $\hat{G}(s)$, it is assumed that the resulting dynamics of the internal loop (depicted as a gray box in Fig. 2) do not follow $G_{D}(s)$ exactly but can be approximately described by a transfer function of the form

$$
G_{I}(s)=\frac{Y(s)}{W(s)}=\frac{b_{I}}{s+a_{I}}
$$

with $G_{I}(s) \neq G_{D}(s)$, where the subscript $I$ stands for "internal loop." If $a_{I}, b_{I}$ were known, the external control law could be designed as

$$
W(s)=\theta_{1} R(s)-\theta_{2} Y(s)
$$

with the controller parameters $\theta_{1}, \theta_{2}$ given by

$$
\theta_{1} \frac{b_{D}}{b_{I}}, \theta_{2}=\frac{a_{D}-a_{I}}{b_{I}}
$$

Indeed, from (4)-(6), it follows that $Y(s) / R(s)=b_{D} /\left(s+a_{D}\right)$, which is the desired transfer function (3). However, since $a_{I}, b_{I}$ are not known beforehand, some adaptive strategy is required to obtain the external

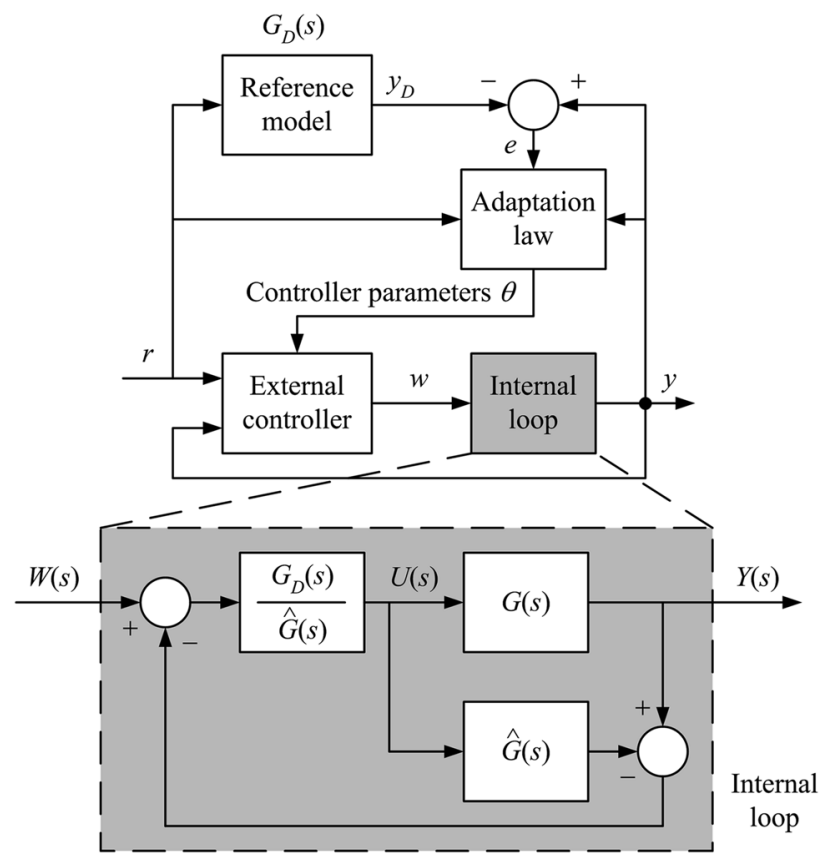

FIG. 2. Control topology including an internal IMC loop and an external MRAC loop. controller parameters $\theta_{1}, \theta_{2}$. Herein, the well-known MIT rule ${ }^{92}$ was employed for this purpose, as described in the Appendix. A unit step reference signal $r(t)$ will be used throughout.

\section{PRELIMINARY EXAMPLE}

This example is concerned with a fractional order system with dynamics described by the following transfer function:

$$
G(s)=\frac{6 s^{1.7}+1}{s^{2.5}+4 s^{0.8}+3} .
$$

The system in (7) displays a damped oscillatory response, which is typical of many feedback-controlled transduction processes encountered in cantilever positioning in atomic force microscopy, ${ }^{93}$ optical force feedback microphones, ${ }^{94-96}$ or the control of complex deexcitation lifetimes encountered in many types of spectroscopies, e.g., nuclear magnetic, ${ }^{97}$ electron-spin, ${ }^{98,99}$ microwave, ${ }^{100-102}$ and multiphoton fluorescence, e.g., Förster resonance, ${ }^{103}$ and in lock-in applications ${ }^{104}$ or in other control and identification schemes. ${ }^{105}$

The desired closed-loop dynamics are specified in the form (6) with $a_{D}=b_{D}=0.5$, which corresponds to a first-order transfer function with a time constant of $2 \mathrm{~s}$. All the simulations were carried out by using the Matlab ${ }^{\circledR} /$ Simulink ${ }^{\circledR}$ software and the FOTF code ${ }^{106}$ for fractional-order systems available within the FOMCON toolbox (www.fomcon.net).

Figure 3 presents the resulting closed-loop response obtained by using the IMC scheme, in the absence of model mismatch, i.e., with $\hat{G}(s)=G(s)$. As can be seen, the actual system output follows the desired response exactly. For comparison, the open-loop response of the simulation model is presented in the inset.

To investigate the effect of model mismatch, possibly arising from an identification process, the simulation was repeated after changing the coefficients and exponents of the simulation model $G(s)$, while keeping the same internal model $\hat{G}(s)$. For this purpose,

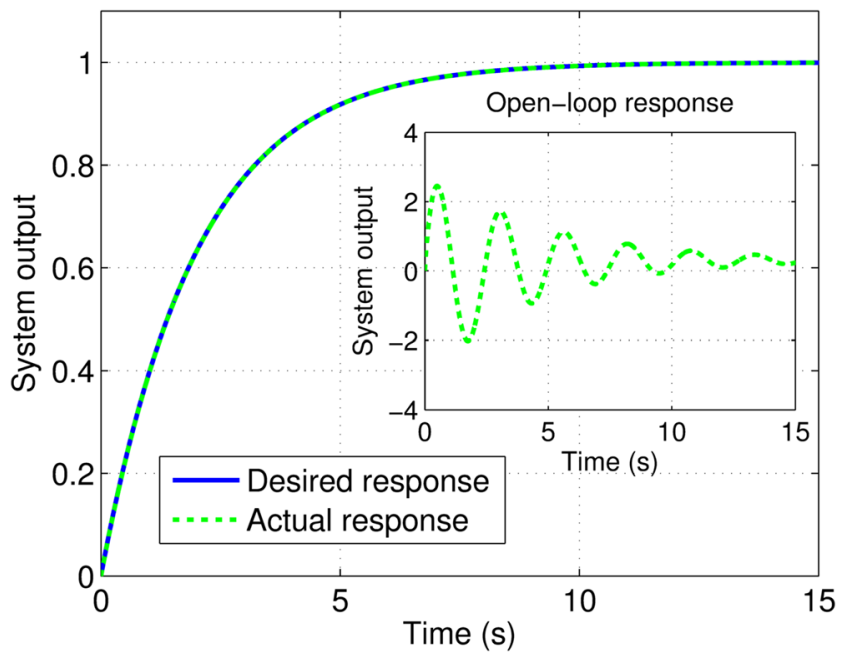

FIG. 3. Closed loop response: fractional-order IMC control scheme with no mode mismatch. The open-loop response is presented in the inset. 

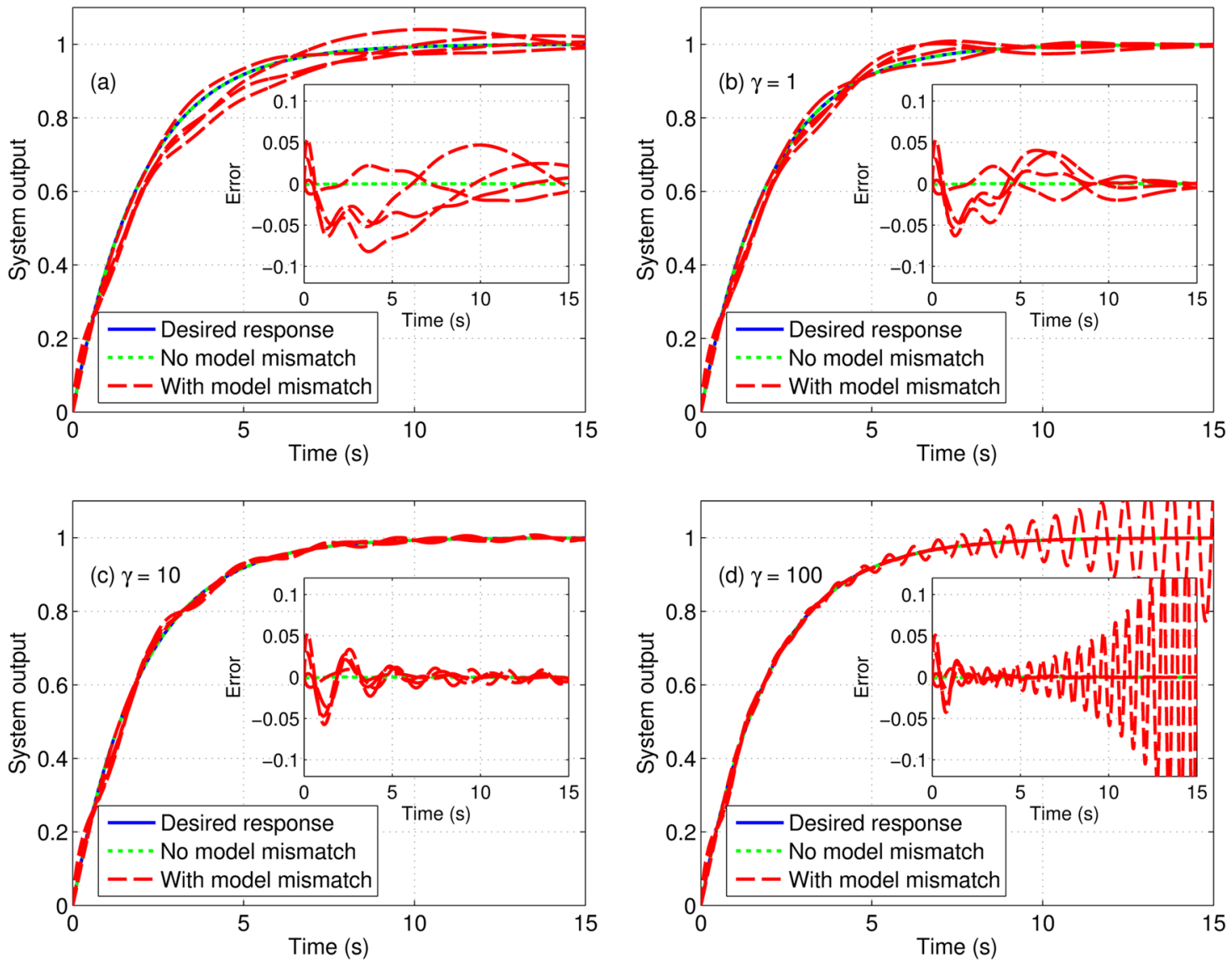

FIG. 4. Closed loop response: (a) fractional-order IMC control scheme with model mismatch. [(b)-(d)] Fractional-order IMC control augmented with the MRAC adaptive contro component using adaptation coefficient $\gamma=1, \gamma=10$, and $\gamma=100$, respectively. The error between the actual responses and the desired response is presented in the insets.

each of the coefficients and exponents was multiplied by a random factor of $(1+v)$, with the value of $v$ extracted from a Gaussian distribution of zero mean and standard deviation of 0.05 . The results of four different simulations are presented in Fig. 4(a). In order to

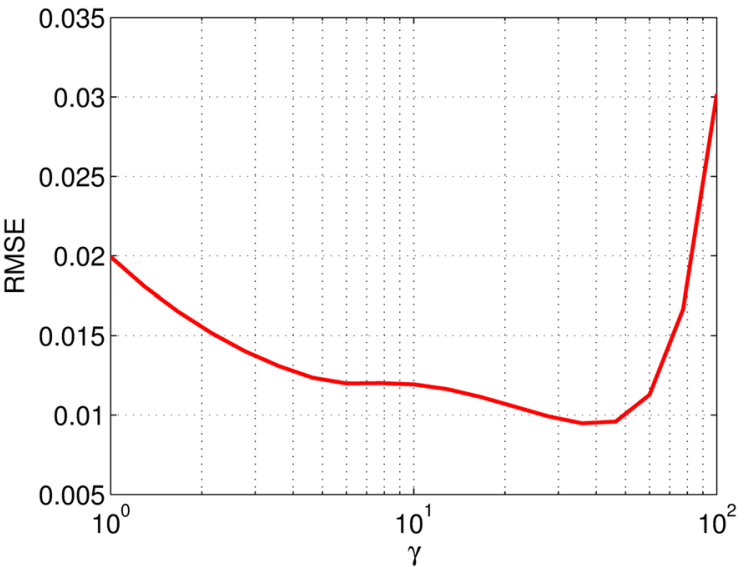

FIG. 5. Fractional-order IMC augmented with the MRAC adaptive control component, in the presence of model mismatch: Root-Mean-Square Error (RMSE) as a function of the adaptation coefficient $\gamma$. mitigate the effect of model mismatch, the external MRAC adaptive control component was employed. The resulting closed-loop responses obtained with the adaptation coefficient (as described in the Appendix) set to $\gamma=1, \gamma=10$, and $\gamma=100$ are shown in Figs. 4(b) $-4(\mathrm{~d})$, respectively. As can be seen, as the value of $\gamma$ is increased, the error with respect to the desired response displays a faster convergence to zero compared to Fig. 4(a). However, a tradeoff is involved because setting $\gamma$ to 100 caused closed-loop instability in one of the simulated cases. This trade-off is illustrated in Fig. 5, which presents the root-mean-square error (RMSE) as a function of the adaptation coefficient $\gamma$. For each value of $\gamma$, the RMSE value was obtained by carrying out four simulations with model mismatch and averaging the square error over time and over the four simulations. The results are presented in Fig. 4. The use of increasingly larger values of $\gamma$ leads to better tracking of the reference signal, as indicated by a reduction in RMSE, up to the point where instability occurs.

\section{APPLICATION TO THE 3D-RC NETWORK}

This application example involves a 3D-RC network with the topology depicted in Fig. 6, comprising 100 resistors and 100 capacitors, in addition to a resistor representing the output resistance of the external voltage source. The resistance and capacitance values were 


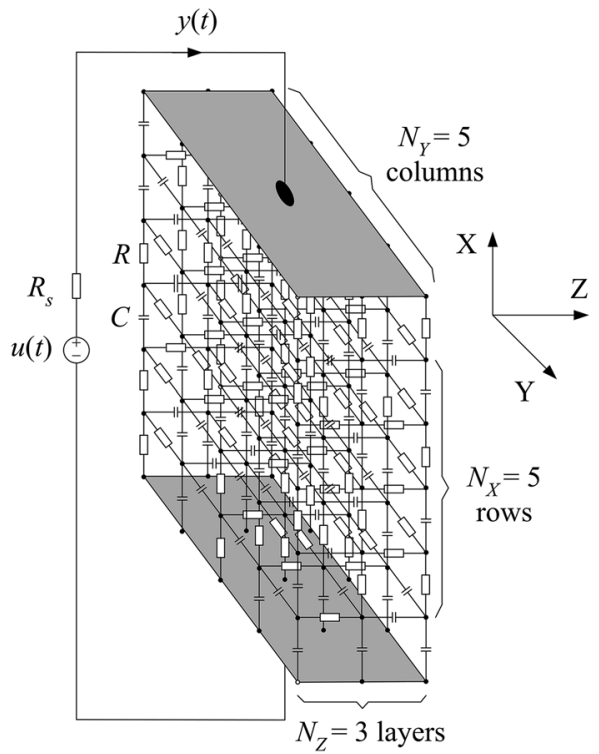

FIG. 6. Three-dimensional RC network connected to a voltage source by the gray conductive plates.

set to $R_{S}=0.1 \Omega, R=1 \Omega$, and $C=0.5 \mathrm{~F}$. This network was employed in Ref. 91 to illustrate an identification method aimed at obtaining fractional-order models on the basis of the measured response to a step input excitation. By taking the voltage $u(t)$ and the current $y(t)$ as input and output signals, respectively, the transfer function $G(s)=Y(s) / U(s)$ corresponds to the network admittance. A detailed analysis of the admittance features in the frequency domain can be found in Ref. 107.

As reported in Ref. 91, a good approximation of the network admittance (including the source resistance $R_{S}$ ) can be achieved by adopting a fractional-order model of the form

$$
\hat{G}(s)=\frac{b_{0}+b_{1} s^{\alpha}}{1+a_{1} s^{\alpha}},
$$

where $b_{0}, b_{1}, a_{1}$ are real-valued coefficients and $\alpha>0$ is a realvalued exponent. The same exponent is employed in the numerator and denominator of (8) because the frequency-domain admittance $\hat{G}(j \omega)$ converges to $1 / R_{S}$ at high frequencies, owing to the presence of capacitor paths between the $3 \mathrm{D}$ network terminals.

By using a step-input identification method, the following transfer function was obtained in Ref. 91:

$$
\hat{G}(s)=\frac{0.287+1.348 s^{0.682}}{1+0.145 s^{0.682}} .
$$

A comparison with the use of an integer-order internal model can be carried out by using the following transfer function:

$$
\hat{G}(s)=\frac{0.358+1.449 s}{1+0.229 s},
$$

which is the identification result reported in Ref. 91 with $\alpha$ set to 1 in the transfer function (8).

Herein, the desired closed-loop dynamics are specified in the form (6) with $a_{D}=b_{D}=10$, which corresponds to a first-order

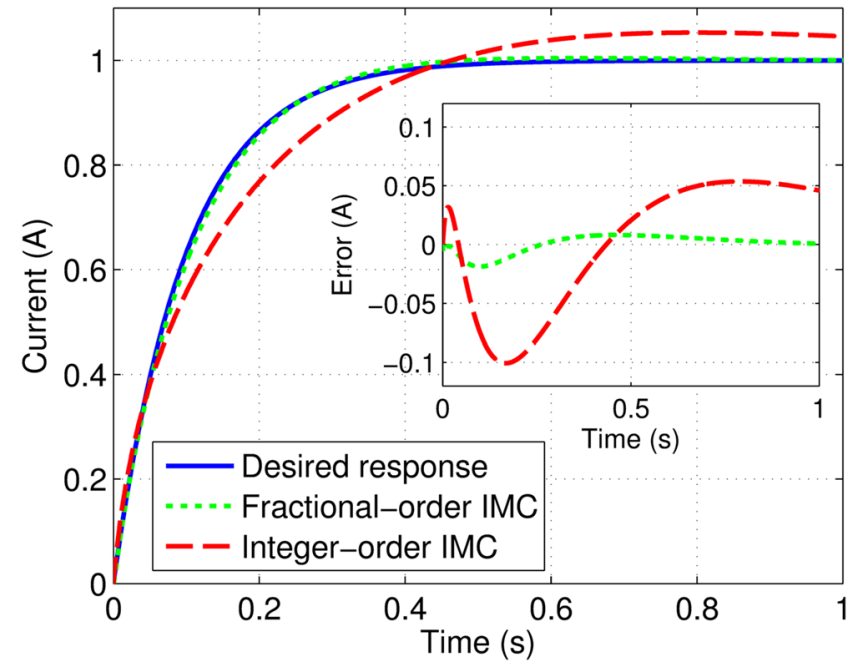

FIG. 7. Closed-loop response: comparison between fractional and integer-order IMC. The error between the actual response and the desired response is presented in the inset.

transfer function with a time constant of $0.1 \mathrm{~s}$. Figure 7 presents the resulting closed-loop responses. As can be seen, the output of the closed-loop system (dotted line) closely follows the desired profile (solid line) if the fractional-order transfer function (9) is employed in the internal model control scheme. The small gap between the solid and dotted lines can be ascribed to minor differences between the fractional-order model (9) and the actual network admittance, as discussed in Ref. 91. In contrast, if the integer-order transfer function (10) is employed, the resulting closed-loop response (dashed line) presents substantially larger deviations from the desired profile. As can be seen in the inset of Fig. 7, the use of a fractional-order model in the IMC scheme does lead to a faster convergence of the error to zero.

In order to study the robustness of the fractional-order IMC controller, the simulation was repeated by using four different $3 \mathrm{D}$ RC networks with random allocation of the R, C components, while keeping the same internal model. As discussed in Ref. 108, this random allocation may result from the natural variability in a production process and results in changes of the network response to electrical excitations. Figure 8(a) presents the resulting closedloop responses. As can be seen, the responses (dashed lines) remain similar to the desired profile (solid line) but with a larger discrepancy compared to the nominal case (dotted line). This discrepancy is reduced by using the external MRAC loop with increasing values of the adaptation coefficient $\gamma$, as shown in Figs. 8(b) and 8(c) for $\gamma=10$ and $\gamma=100$, respectively. However, setting $\gamma$ to 1000 caused closed-loop instability in two of the simulated cases, as seen in Fig. 8(d). This trade-off is illustrated in Fig. 9, which presents the root-mean-square error (RMSE) as a function of $\gamma$. As in the preliminary example presented in Sec. IV, the use of increasingly larger values of $\gamma$ leads to a reduction in RMSE up to the point where instability occurs.

Additional simulations were carried out to investigate the effect of measurement noise. For this purpose, at each time step of the 

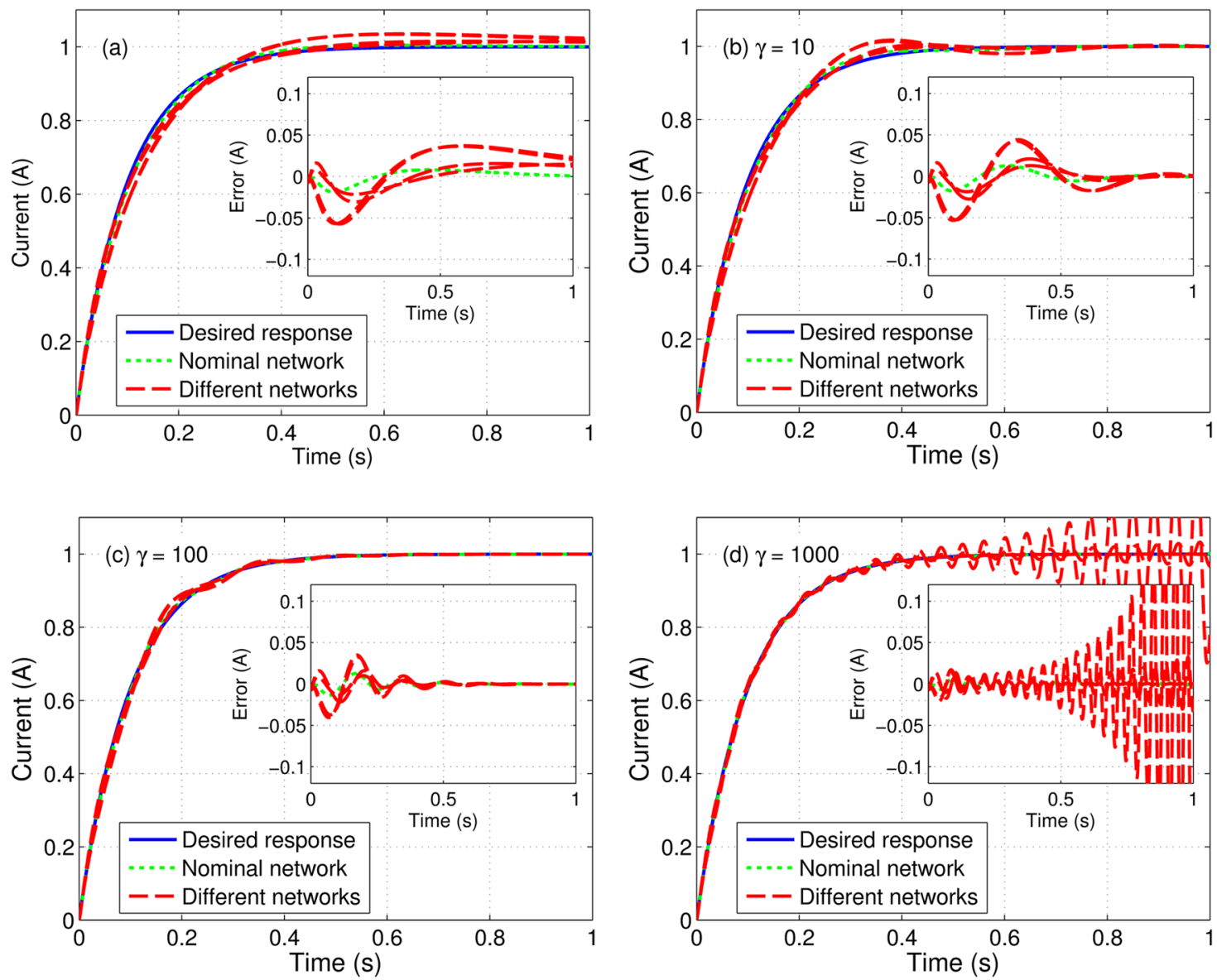

FIG. 8. Closed-loop response: comparison between the nominal network and four different networks with random allocation of the R, C components. (a) Fractional-order IMC control scheme. [(b)-(d)] Fractional-order IMC control augmented with the MRAC adaptive control component using adaptation coefficient $\gamma=10, \gamma=100$, and $\gamma=1000$. The error between the actual responses and the desired response is presented in the insets.

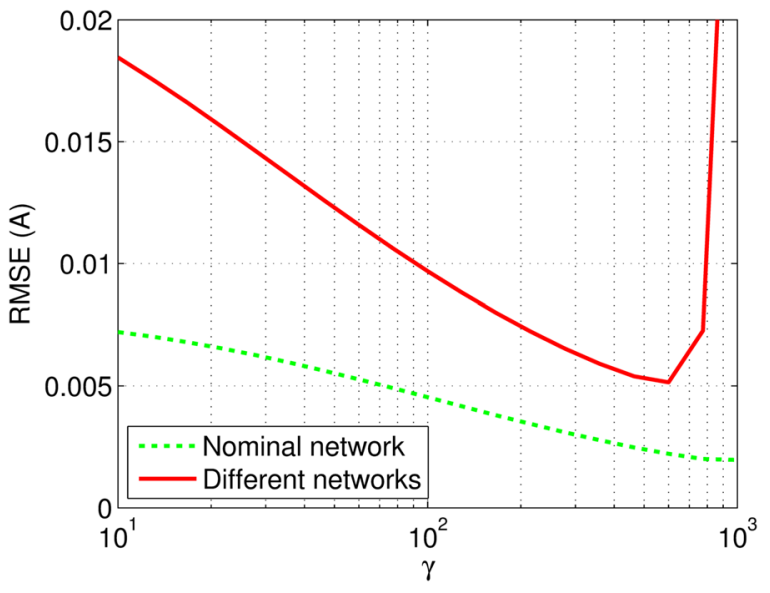

FIG. 9. Fractional-order IMC augmented with the MRAC adaptive control component: Root-Mean-Square Error (RMSE) as a function of the adaptation coefficient $\gamma$. The vertical axis is clipped for better visualization. simulation procedure, the feedback data were corrupted with random noise from a Gaussian distribution of zero mean and standard deviation of 0.02 . The results are shown in Fig. 10 for the fractional-order IMC scheme without [Fig. 10(a)] and with the MRAC adaptive control component $[\gamma=10$ in Fig. 10(b) and $\gamma=100$ in Fig. $10(\mathrm{c})$ ]. The value $\gamma=1000$ was not employed because it caused instability in the noise-free case, as shown in Fig. 8(d).

By comparing Fig. 10(a) with Figs. 10(b) and 10(c), it can be noted that the use of MRAC still provides a faster reduction in the error magnitude. However, the presence of measurement noise gives rise to oscillations of the response around the reference value, which are more clearly visible for $\gamma=100$ [Fig. 10(c)], compared to $\gamma=10$ [Fig. 10(b)]. Indeed, as shown in Fig. 10(d), owing to the presence of noise, the root-mean-square value of the error starts to increase for larger values of the adaptation coefficient $\gamma$, even in the nominal network case. It is worth noting that the RMSE values corresponding to the solid lines in Figs. 9 and 10 (d) were obtained for each value of $\gamma$ as the square root of the square error averaged over time and over the four different networks. 

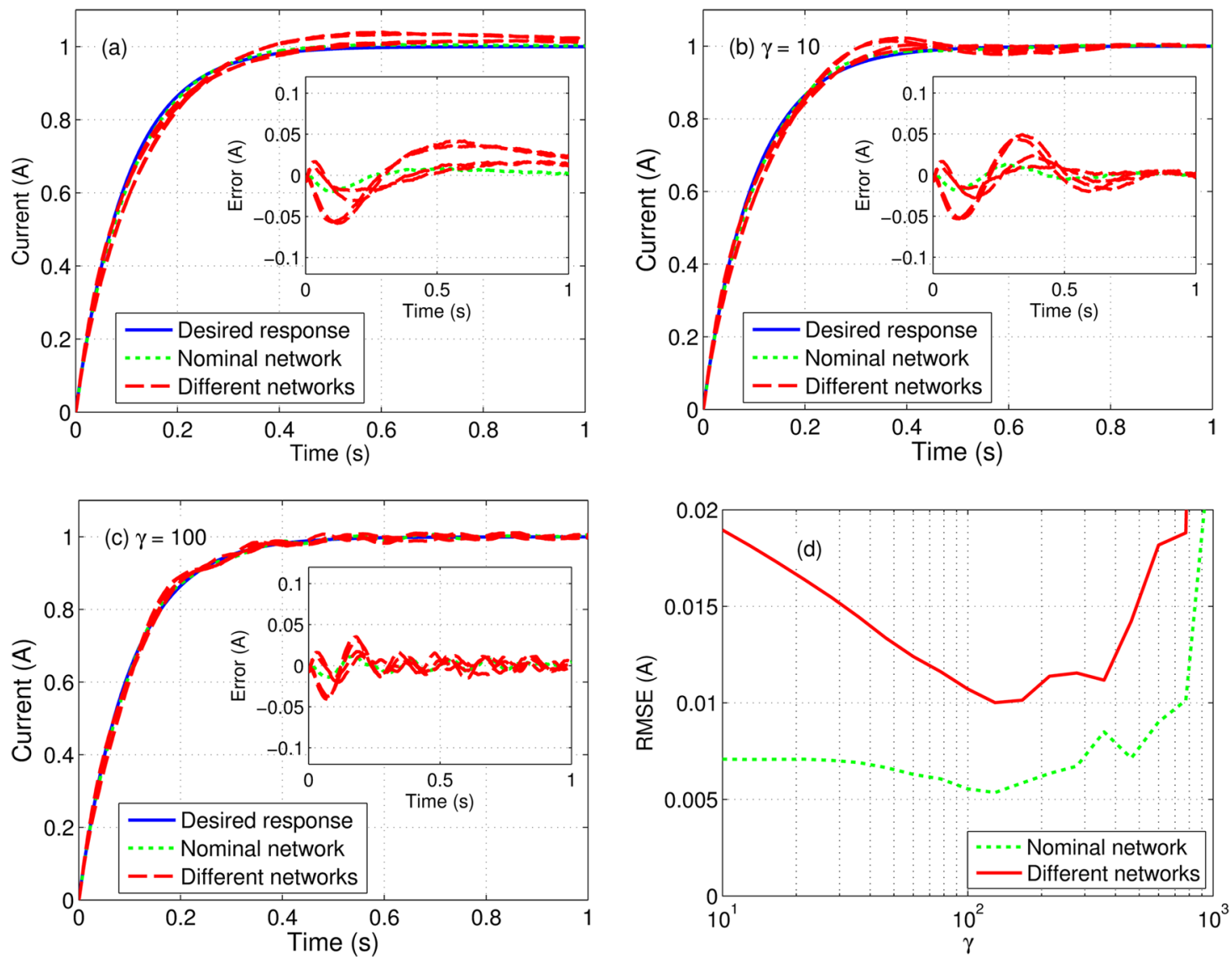

FIG. 10. Closed-loop results in the presence of measurement noise using the fractional-order IMC scheme: (a) without MRAC and [(b) and (c)] with MRAC. The RMSE values are shown in (d), with the vertical axis clipped for better visualization.

\section{CONCLUSION}

A fractional-order internal model control technique is applied to systems described by fractional order dynamics to impose a first order response. Fractional order emergent responses are emulated using three-dimensional RC networks and through an example of a system model with multiple fractional order exponents. The control law is augmented with a model-reference adaptive strategy in an external loop. By imposing a control law on the system to obey first order dynamics, a calibrated transient response is ensured. The MRAC scheme provides additional robustness to model mismatch from the identification process and faster error convergence to zero. The proposed methodology enables feedback control of complex systems with emergent responses and is thus of value to a wide range of transduction processes with emergent dynamics encountered in feedback instrumentation, e.g., in electronic, microwave, and photonic lock-in problems. Finally, there are further applications across the broader measurement science and instrumentation community designing complex feedback systems at the system level, e.g., ensuring the adaptive control of distributed physiological processes through the use of biomedical implants that would be capable of responding to fractional order dynamics. Future work may also investigate the possibility of extending this study to complex systems emulated using a large-scale three-dimensional RLC network. ${ }^{109}$

\section{ACKNOWLEDGMENTS}

This work was supported by Fundação de Amparo à Pesquisa do Estado de São Paulo (Grant Nos. 2011/17610-0 and 2011/137778) and Conselho Nacional de Desenvolvimento Científico e Tecnológico (Grant Nos. 303714/2014-0 and 303393/2018-1).

\section{APPENDIX: MIT RULE}

Let $y_{D}$ be the output of the reference model, as indicated in Fig. 2. Moreover, let $e=y-y_{D}$ be the error between the actual system response $y$ and the desired response $y_{D}$. At each time instant, a cost function $J$ associated with the controller parameters $\theta_{1}, \theta_{2}$ can be defined as

$$
J\left(\theta_{1}, \theta_{2}\right)=\frac{1}{2} e^{2} .
$$

The MIT rule consists in adjusting the controller parameters in a gradient-descent manner, ${ }^{92}$ i.e.,

$$
\dot{\theta}_{1}=-\gamma^{\prime} \frac{\partial J}{\partial \theta_{1}}=-\gamma^{\prime} e \frac{\partial e}{\partial \theta_{1}}=-\gamma^{\prime} e \frac{\partial y}{\partial \theta_{1}}
$$


where $\gamma^{\prime}>0$ is a constant adaptation coefficient. In a similar manner, $\theta_{2}$ is adapted as

$$
\dot{\theta}_{2}=-\gamma^{\prime} e \frac{\partial y}{\partial \theta_{2}} .
$$

From (4) and (5), it follows that

$$
y=\frac{b_{I} \theta_{1}}{p+a_{I}+b_{I} \theta_{2}} r
$$

where $p$ is the time-differential operator, following the notation adopted in Ref. 92. Therefore, the partial derivatives of the error $e$ in (A2) and (A3) can be calculated as

$$
\begin{gathered}
\frac{\partial y}{\partial \theta_{1}}=\frac{b_{I}}{p+a_{I}+b_{I} \theta_{2}} r, \\
\frac{\partial y}{\partial \theta_{2}}=\frac{-b_{I}^{2} \theta_{1}}{\left(p+a_{I}+b_{I} \theta_{2}\right)^{2}} r=\frac{-b_{I}}{p+a_{I}+b_{I} \theta_{2}} y,
\end{gathered}
$$

where the last identity follows from (A4). Now, assuming that the closed-loop response (A4) will be approximately equal to the desired response $(3)$, one may write $a_{I}+b_{I} \theta_{2} \approx a_{D}$. By using this approximation in (A5) and (A6), the adaptation laws (A2) and (A3) become

$$
\begin{gathered}
\dot{\theta}_{1}=-\gamma^{\prime} \frac{b_{I}}{p+a_{D}} r=-\gamma e \frac{a_{D}}{p+a_{D}} r, \\
\dot{\theta}_{2}=\gamma e \frac{a_{D}}{p+a_{D}} y,
\end{gathered}
$$

where $\gamma=\gamma^{\prime} b_{I} / a_{D}$. By doing so, the unknown coefficient $b_{I}$ is incorporated into a new adaptation coefficient $\gamma$, which becomes a design parameter in the resulting MRAC law. In the present work, it is assumed that $\left(a_{I}, b_{I}\right)$ are close to $\left(a_{D}, b_{D}\right)$ due to the action of the internal IMC control loop. Therefore, in view of (6), the external controller parameters were initialized as $\theta_{1}=1$ and $\theta_{2}=0$.

\section{REFERENCES}

${ }^{1}$ L. Shalalfeh, P. Bogdan, and E. Jonckheere, "Evidence of long-range dependence in power grid," in 2016 IEEE Power and Energy Society General Meeting (PESGM), Boston, MA (IEEE, 2016), pp. 1-5.

${ }^{2}$ J. Sia, E. Jonckheere, L. Shalalfeh, and P. Bogdan, "PMU change point detection of imminent voltage collapse and stealthy attacks," in 2018 IEEE Conference on Decision and Control (CDC), Miami Beach, FL, 2018 (IEEE, 2018), pp. 6812-6817. ${ }^{3}$ M. E. Fouda, A. S. Elwakil, A. G. Radwan, and A. Allagui, "Power and energy analysis of fractional-order electrical energy storage devices," Energy 111, 785-792 (2016).

${ }^{4}$ S. P. Jadhav, R. H. Chile, and S. T. Hamde, "Robust fractional-order controller using Bode's ideal transfer function for power plant gas turbine," Int. J. Comput. Appl. 88, 1-7 (2014).

${ }^{5}$ C. Abbey and G. Joos, "Supercapacitor energy storage for wind energy applications,” IEEE Trans. Ind. Appl. 43, 769-776 (2007).

${ }^{6}$ R. Martin and J. Quintana, "Modeling of electrochemical double layer capacitors by means of fractional impedance," J. Comput. Nonlinear Dyn. 3, 021303 (2008).

${ }^{7}$ S. Buller, E. Karden, D. Kok, and R. Doncker, "Modeling the dynamic behaviour of supercapacitors using impedance spectroscopy," IEEE Trans. Ind. Appl. 38, $1622-1626$ (2002)

${ }^{8}$ H. Samavati, A. Hajimiri, A. Shahani, G. Nasserbakht, and T. Lee, "Fractal capacitors," IEEE J. Solid-State Circuits 33, 2035-2041 (1998).

${ }^{9}$ C. Psychalinos, A. S. Elwakil, B. J. Maundy, and A. Allagui, "Analysis and realization of a switched fractional-order-capacitor integrator," Int. J. Circuit Theory Appl. 44, 2035-2040 (2016).
${ }^{10}$ A. S. Elwakil, A. G. Radwan, T. Freeborn, A. Allagui, B. J. Maundy, and M. Fouda, "Low-voltage commercial super-capacitor response to periodic linearwith-time current excitation: A case study," IET Circuit Device Syst. 11, 189-195 (2016).

${ }^{11}$ B. Wang, Z. Liu, S. E. Li, S. J. Moura, and H. Peng, "State-of-charge estimation for lithium-ion batteries based on a nonlinear fractional model," IEEE Trans. Control Syst. Technol. 25, 3-11 (2017).

${ }^{12}$ A. S. Elwakil, "Fractional-order circuits and systems: An emerging interdisciplinary research area," IEEE Circuits Syst. Mag. 10, 40-50 (2010).

${ }^{13}$ A. D. Freed and K. Diethelm, "Fractional calculus in biomechanics: A 3D viscoelastic model using regularized fractional derivative kernels with application to the human calcaneal fat pad," Biomech. Model. Mechanobiol. 5, 203-215 (2006).

${ }^{14}$ D. Craiem and R. Armentano, "A fractional derivative model to describe arterial viscoelasticity,” Biorheology 44, 251-263 (2007).

${ }^{15} \mathrm{C}$. Ionescu and R. De Keyser, "Parametric models for characterizing respiratory input impedance,” J. Med. Eng. Technol. 32, 315-324 (2008).

${ }^{16}$ C. Ionescu and R. De Keyser, "Relations between fractional-order model parameters and lung pathology in chronic obstructive pulmonary disease," IEEE Trans. Biomed. Eng. 56, 978-987 (2009).

${ }^{17}$ R. Magin, X. Feng, and D. Baleanu, "Solving the fractional order Bloch equation concepts,” Concepts Magn. Reson., Part A 34, 16-23 (2009).

${ }^{18}$ M. Ghorbani and P. Bogdan, "A cyber-physical system approach to artificial pancreas design," in 2013 International Conference on Hardware/Software Codesign and System Synthesis (CODES+ISSS), Montreal, QC (IEEE Press, 2013), Article No. 17, pp. 1-10.

${ }^{19}$ S. Pequito, P. Bogdan, and G. J. Pappas, "Minimum number of probes for brain dynamics observability," in 2015 54th IEEE Conference on Decision and Control (CDC), Osaka (IEEE, 2015), pp. 306-311.

${ }^{20} \mathrm{M}$. Ghorbani and P. Bogdan, "Reducing risk of closed loop control of blood glucose in artificial pancreas using fractional calculus," in 2014 36th Annual International Conference of the IEEE Engineering in Medicine and Biology Society, Chicago, IL (IEEE, 2014), pp. 4839-4842.

${ }^{21}$ G. Gupta, S. Pequito, and P. Bogdan, "Re-thinking EEG-based non-invasive brain interfaces: Modeling and analysis," in 2018 ACM/IEEE 9th International Conference on Cyber-Physical Systems (ICCPS), Porto (IEEE, 2018), pp. 275-286.

${ }^{22}$ T. J. Freeborn, "A survey of fractional-order circuit models for biology and biomedicine,” IEEE J. Emerging Sel. Top. Circuits Syst. 3, 416-423 (2013).

${ }^{23}$ K. S. Cole and R. H. Cole, "Dispersion and absorption in dielectrics: Alternating current characteristics," J. Chem. Phys. 9, 341-351 (1941).

${ }^{24}$ B. Rigaudt, L. Hamzaouit, M. Frikhaf, N. Chauveaut, and J. Moruccit, "In vitro tissue characterization and modelling using electrical impedance measurements in the $100 \mathrm{~Hz}-10 \mathrm{MHz}$ frequency range," Physiol. Meas. 16, A15-A28 (1995).

${ }^{25}$ A. S. Elwakil and B. Maundy, "Extracting the Cole-Cole impedance model parameters without direct impedance measurement," Electron. Lett. 46, 1367-1368 (2010).

${ }^{26}$ B. Maundy and A. S. Elwakil, "Extracting single dispersion Cole-Cole impedance model parameters using an integrator setup," Analog Integr. Circuits Signal Process. 71, 107-110 (2012).

${ }^{27}$ T. J. Freeborn, B. Maundy, and A. S. Elwakil, "Cole impedance extractions from the step-response of a current excited fruit sample," Comput. Electron. Agric. 98, 100-108 (2013).

${ }^{28}$ S. Hadjiloucas, G. C. Walker, J. W. Bowen, and R. K. H. Galvão, "System identification algorithms for the analysis of dielectric responses from broadband spectroscopies,” J. Phys.: Conf. Ser. 310, 012002 (2011).

${ }^{29}$ I. Petráš, "Fractional-order memristor-based Chua's circuit," IEEE Trans. Circuits Syst. II Express Briefs 57, 975-979 (2010).

${ }^{30}$ W. T. Coffey, D. A. Garanin, and D. J. McCarthy, "Crossover formulas in the Kramers theory of thermally activated escape rates-Application to spin systems," Adv. Chem. Phys. 117, 483 (2001).

${ }^{31}$ N. Wei, P. M. Déjardin, Yu. P. Kalmykov, and W. T. Coffey, "External dc biasfield effects in the nonlinear ac stationary response of dipolar particles in a meanfield potential," Phys. Rev. E 93, 042208 (2016).

${ }^{32}$ P. M. Déjardin and F. Ladieu, "Nonlinear susceptibilities of interacting polar molecules in the self-consistent field approximation," J. Chem. Phys. 140, 034506 (2014). 
${ }^{33}$ P. M. Déjardin, S. V. Titov, and Y. Cornaton, "Linear complex susceptibility of long-range interacting dipoles with thermal agitation and weak external ac fields," Phys. Rev. B 99, 024304 (2019)

${ }^{34} \mathrm{M}$. Ortigueira and J. Machado, "Special issue on fractional calculus applications in signals and systems," Signal Process. 86, 2503-2504 (2006).

${ }^{35} \mathrm{G}$. Maione, "Closed-form rational approximations of fractional, analog and digital differentiators/integrators," IEEE Trans. Emerging Sel. Top. Circuits Syst. 3, 322-329 (2013).

${ }^{36}$ R. J. M. Afonso, R. K. H. Galvão, K. H. Kienitz, H. Alyami, V. M. Becerra, and S. Hadjiloucas, "Constrained pre-equalization accounting for multi-path fading emulated using large RC networks: Applications to wireless and photonics communications," Opt. Quantum Electron. 48, 1-20 (2016).

${ }^{37} \mathrm{M}$. Lupupa and S. Hadjiloucas, "Fractional-order system identification in massive MIMO systems,” J. Phys.: Conf. Ser. 1065, 212016 (2018).

${ }^{38}$ O. Cois, A. Oustaloup, T. Poinot, and J.-L. Battaglia, "Fractional state variable filter for system identification by fractional model," in 2001 European Control Control Conference, (ECC), Porto Portugal (IEEE, 2001), pp. 2481-2486.

${ }^{39}$ T. T. Hartley and C. F. Lorenzo, "Fractional-order system identification based on continuous order-distributions," Signal Process. 83, 2287-2300 (2003).

${ }^{40}$ A. Tepljakov, E. Petlenkov, and J. Belikov, "FOMCON: Fractional-order modeling and control toolbox for MATLAB," in Proceedings of the 18th International Conference Mixed Design of Integrated Circuits and Systems-MIXDES 2011 (IEEE, 2011), pp. 684-689.

${ }^{41}$ L. Dorčák, V. Leško, and I. Koštial, "Identification of fractional order dynamical systems," preprint arXiv:math/0204187 (2002).

${ }^{42}$ D.-Y. Liu, T.-M. Laleg-Kirati, O. Olivier Gibaru, and W. Perruquetti, "Identification of fractional order systems using modulating functions method," in 2013 American Control Conference (ACC), Washington, DC, USA (IEEE, 2013), pp. 1679-1684.

${ }^{43}$ S. Hadjiloucas and R. K. H. Galvão, "Fractional order system identification algorithms for biomedical applications," J. Phys.: Conf. Ser. 490, 012141 (2014).

${ }^{44}$ Y. Xue and P. Bogdan, "Constructing compact causal mathematical models for complex dynamics," in Proceedings of the 8th International Conference on CyberPhysical Systems (ICCPS '17) (ACM, New York, NY, USA, 2017), pp. 97-107.

${ }^{45} \mathrm{G}$. Gupta, S. Pequito, and P. Bogdan, "Dealing with unknown unknowns: Identification and selection of minimal sensing for fractional dynamics with unknown inputs," in 2018 Annual American Control Conference (ACC), Milwaukee, WI (IEEE, 2018), pp. 2814-2820.

${ }^{46}$ A. G. Radwan and K. N. Salama, "Fractional-order RC and RL circuits," Circuits, Syst., Signal Process. 31, 1901-1915 (2012).

${ }^{47}$ A. G. Radwan, A. S. Elwakil, and A. M. Soliman, "Fractional-order sinusoidal oscillators: Design procedure and practical examples," IEEE Trans. Circuits Syst. I: Regular Pap. 55, 2051-2063 (2008).

${ }^{48}$ A. G. Radwan, A. S. Elwakil, and A. M. Soliman, "On the generalization of second-order filters to the fractional-order domain," J. Circuits Syst. Comput. 18, 361-386 (2009).

${ }^{49}$ D. Mondal and K. Biswas, "Performance study of fractional order integrator using single-component fractional order element," IET Circuits Devices Syst. 5, 334-342 (2011).

${ }^{50}$ B. Maundy, A. S. Elwakil, and T. J. Freeborn, "On the practical realization of higher-order filters with fractional stepping," Signal Process. 91, 484-491 (2011).

${ }^{51}$ A. G. Radwan, "Resonance and quality factor of the $\mathrm{RL}^{\mathrm{a}} \mathrm{C}^{\mathrm{a}}$ fractional circuit," IEEE J. Emerging Sel. Top. Circuits Syst. 3, 377-385 (2013).

${ }^{52}$ M. S. Sarafraz and M. S. Tavazoei, "Realizability of fractional-order impedances by passive electrical networks composed of a fractional capacitor and RLC components," IEEE Trans. Circuits Syst. I: Regular Pap. 62, 2829-2835 (2015).

${ }^{53}$ M. E. Fouda, A. S. Elwakil, A. G. Radwan, and B. J. Maundy, "Fractional-order two-port networks," Math. Probl. Eng. 2016, 5976301.

${ }^{54}$ I. Petráš, "The fractional-order controllers: Methods for their synthesis and application," e-print arXiv:math/0004064v1 [math.OC] (11 April 2000).

${ }^{55}$ I. Podlubny, I. Petráš, B. M. Vinagre, P. O'Leary, and L. Dorčák, "Analogue realizations of fractional-order controllers," Nonlinear Dyn. 29, 281-296 (2002).

${ }^{56} \mathrm{~A}$. Charef, "Analogue realisation of fractional-order integrator, differentiator and fractional $\mathrm{PI}^{\lambda} \mathrm{D}^{\mu}$ controller," IEE Proc.: Control Theory Appl. 153, 714-720 (2006).
${ }^{57}$ Y. Luo and Y.-Q. Chen, "Fractional order [proportional derivative] controller for a class of fractional order systems," Automatica 45, 2446-2450 (2009).

${ }^{58}$ I. Petráš, "Stability of fractional-order systems with rational orders," e-print arXiv:0811.4102v2 [Math.DS] (5 December 2008).

${ }^{59}$ A. G. Radwan, A. M. Soliman, A. S. Elwakil, and A. Sedeek, "On the stability of linear systems with fractional-order elements," Chaos, Solitons Fractals $\mathbf{4 0}$, 2317-2328 (2009).

${ }^{60}$ M. S. Tavazoei and M. Haeri, "Rational approximations in the simulation and implementation of fractional-order dynamics: A descriptor system approach," Automatica 46, 94-100 (2010).

${ }^{61}$ C. E. Garcia and M. Morari, "Internal model control. 1. A unifying review and some new results,” Ind. Eng. Chem. Process Des. Dev. 21, 308 (1982).

${ }^{62}$ M. Morari and E. Zafiriou, Robust Process Control (Prentice-Hall, Englewood Cliffs, NJ, 1989).

${ }^{63}$ R. D. Braatz, Internal Model Control the Control Handbook (CRC Press, Boca Raton, FL, 1995).

${ }^{64}$ C. E. Garcia and M. Morari, "Internal model control. 2. Design procedure for multivariable systems," Ind. Eng. Chem. Process Des. Dev. 24, 472-484 (1985).

${ }^{65}$ C. E. Garcia and M. Morari, "Internal model control. 3. Multivariable control law computation and tuning guidelines," Ind. Eng. Chem. Process Des. Dev. 24, 484-494 (1985).

${ }^{66}$ C. G. Economou, M. Morari, and B. O. Palsson, "Internal model control. 5. Extension to nonlinear systems," Ind. Eng. Chem. Process Des. Dev. 25, 403-411 (1986).

${ }^{67} \mathrm{~K}$. J. Hunt and D. Sbarbaro, "Neural networks for nonlinear internal model control," IEE Proc.-D: Control Theory Appl. 138, 431-438 (1991).

${ }^{68}$ A. Zheng, M. V. Kothare, and M. Morari, "Anti-windup design for internal model control control and dynamical systems," Technical Memorandum No. CIT-CDS93-007, California Institute of Technology, May 1993, pp. 1-11.

${ }^{69}$ J.-P. Calvet and Y. Arkun, "Feedforward and feedback linearization of nonlinear systems and its implementation using internal model control (IMC)," Ind. Eng. Chem. Res. 27, 1822-1831 (1988).

${ }^{70}$ S. Ladaci, J. J. Loiseau, and A. Charef, "Adaptive internal model control with fractional order parameter," Int. J. Adapt. Control Signal Process. 24, 944-960 (2010).

${ }^{71}$ T. Vinobrava, N. Sivakumaran, S. Narayanan, and T. K. Radhakrishnan, "Design of internal model control based fractional order PID controller," J. Control Theory Appl. 10, 297-302 (2012).

${ }^{72}$ S. P. Jadhav, R. H. Chile, and S. T. Hamde, "Modeling and design of fractionalorder IMC based controller for power plant gas turbine," in Proceedings of the ASME 2015 Gas Turbine India Conference (ASME, 2015), p. V001T06A002, Paper No. GTINDIA2015-1264.

${ }^{73} \mathrm{U}$. Al-Saggaf, I. Mehedi, M. Bettayeb, and R. Mansouri, "Fractional-order controller design for a heat flow process," J. Syst. Control Eng. 230, 680-691 (2016).

${ }^{74} \mathrm{~J}$. C. Wang, "Realizations of generalized Warburg impedance with RC ladder networks and transmission lines," J. Electrochem. Soc. 134, 1915-1920 (1987).

${ }^{75}$ S. G. Samko, A. A. Kilbas, and O. I. Marichev, Fractional Integrals and Derivatives-Theory and Applications (Gordon and Breach Science Publishers, New York, 1987).

${ }^{76}$ I. Podlubny, "Geometric and physical interpretation of fractional integration and fractional differentiation," Fractional Calc. Appl. Anal. 5, 367-386 (2002).

${ }^{77}$ D. Cafagna, "Past and present-fractional calculus: A mathematical tool from the past for present engineers," IEEE Ind. Electron. Mag. 1, 35-40 (2007).

${ }^{78} \mathrm{M}$. D. Ortigueira, "An introduction to the fractional continuous-time linear systems: The 21st century systems," IEEE Circuits Syst. Mag. 8, 19-26 (2008).

${ }^{79} \mathrm{~J}$. Mayes and M. Sen, "Approximation of potential-driven flow dynamics in large-scale self-similar tree networks," Proc. R. Soc. A 467, 2810-2824 (2011).

${ }^{80}$ T. Clarke, B. N. N. Achar, and J. W. Hanneken, "Mittag-Leffler functions and transmission lines,” J. Mol. Liq. 114, 159-163 (2004).

${ }^{81}$ P. J. Torvik and R. L. Bagley, "On the appearance of the fractional derivative in the behavior of real materials," J. Appl. Mech. 51, 294-298 (1984).

${ }^{82}$ J. J. De Espíndola, J. M. Da Silva Neto, and E. M. O. Lopes, "A generalized fractional derivative approach to viscoelastic material properties measurement," Appl. Math. Comput. 164, 493-506 (2005). 
${ }^{83}$ J. Sabatier, M. Aoun, A. Oustaloup, G. Gregoire, F. Ragot, and P. Roy, "Fractional system identification for lead acid battery state charge estimation," Signal Process. 86, 2645-2657 (2006)

${ }^{84}$ I. Schäfer and K. Krüger, "Modelling of coils using fractional derivatives," J. Phys. D: Appl. Phys. 41, 045001 (2008).

${ }^{85}$ R. F. Voss and J. Clarke, "1/f noise in speech and music," Nature 258, 317-318 (1975).

${ }^{86}$ M. S. Keshner, “1/f noise,” Proc. IEEE 70, 212-218 (1982).

${ }^{87}$ M. Caputo, "Distributed order differential equations modeling dielectric induction and diffusion," Fractional Calc. Appl. Anal. 4, 421-442 (2001).

${ }^{88}$ R. Gorenflo and F. Mainardi, in Fractional Diffusion Processes: Probability Distributions and Continuous Time Random Walk in Long Range Dependent Processes: Theory and Applications, edited by G. Rangarajan and M. Ding (SpringerVerlag, New York, 2003).

${ }^{89}$ M. D. Ortigueira and A. G. Batista, "On the relation between the fractional Brownian motion and the fractional derivatives," Phys. Lett. A 372, 958-968 (2008).

${ }^{90}$ G. F. Franklin, J. D. Powell, and A. Emami-Naeini, Feedback Control of Dynamic Systems, 5th ed. (Prentice-Hall, Upper Saddle River, 2006).

${ }^{91}$ L. A. Jacyntho, M. C. M. Teixeira, E. Assunção, R. Cardim, R. K. H. Galvão, and S. Hadjiloucas, "Identification of fractional-order transfer functions using a step excitation,” IEEE Trans. Circuits Syst. II: Express Briefs 62, 896-900 (2015).

${ }^{92}$ K. J. Astrom and B. Wittenmark, Adaptive Control, 2nd ed. (Addison-Wesley, Boston, 1994).

${ }^{93}$ F. J. Giessibl, "Advances in atomic force microscopy," Rev. Mod. Phys. 75, 949-983 (2003).

${ }^{94}$ L. S. Karatzas, D. A. Keating, and M. J. Usher, “A practical optical force-feedback microphone,” Trans. Inst. Meas. Control 16, 75 (1994).

${ }^{95}$ S. Hadjiloucas, L. S. Karatzas, D. A. Keating, and M. J. Usher, "Optical sensors for monitoring water uptake in plants," J. Lightwave Technol. 13, 1421-1428 (1995).

${ }^{96}$ S. Hadjiloucas, G. Walker, J. Bowen, and L. S. Karatzas, “An optical forcefeedback microphone for sensing biophotonics related photoacoustic and photothermal phenomena," in EOS Annual Meeting, 2008, Paris, France (European Optical Society, 2008), p. 968, ISBN: 978-3-00-024188-8.

${ }^{97}$ V. Calandrini, D. Abergel, and G. R. Kneller, "Fractional protein dynamics seen by nuclear magnetic resonance spectroscopy: Relating molecular dynamics simulation and experiment,” J. Chem. Phys. 133, 145101 (2010).
${ }^{98}$ L. Tiemann, G. Gamez, N. Kumada, and K. Muraki, "Unraveling the spin polarization of the $v=5 / 2$ fractional quantum Hall state," Science 335, 828-831 (2012).

${ }^{99}$ V. Vitzthum, F. Borcard, S. Jannin, M. Morin, P. Miéville, M. A. Caporini, A. Sienkiewicz, S. Gerber-Lemaire, and G. Bodenhausen, "Fractional spin-labeling of polymers for enhancing NMR sensitivity by solvent-free dynamic nuclear polarization," Chem. Phys. Chem. 12, 2929-2932 (2011).

${ }^{100}$ I. A. Dmitriev, A. D. Mirlin, and D. G. Polyakov, "Theory of fractional microwave-induced resistance oscillations," Phys. Rev. Lett. 99, 206805 (2007).

${ }^{101}$ S. Wiedmann, G. M. Gusev, O. E. Raichev, A. K. Bakarov, and J. C. Portal, "High-order fractional microwave-induced resistance oscillations in twodimensional systems," Phys. Rev. B 80, 035317 (2009).

${ }^{102}$ A. A. Aligia and L. Arrachea, "Entangled end states with fractionalized spin projection in a time-reversal-invariant topological superconducting wire," Phys. Rev. B 98, 174507 (2018); e-print arXiv:1806.06104.

${ }^{103}$ P. R. Barber, S. M. Ameer-Beg, J. Gilbey, L. M. Carlin, M. Keppler, T. C. $\mathrm{Ng}$, and B. Vojnovic, "Multiphoton time-domain fluorescence lifetime imaging microscopy: Practical application to protein-protein interactions using global analysis,"J. R. Soc. Interfaces 6 (2008).

${ }^{104}$ R. El-Khazali, "Fractional-order digital phase-locked loop," in 14th IEEE International Conference on Electronics, Circuits and Systems (IEEE, 2007), pp. 963-966.

${ }^{105}$ M. Aoun, R. Malti, F. Levron, and A. Oustaloup, "Synthesis of fractional Laguerre basis for system approximation," Automatica 43, 1640-1648 (2007).

${ }^{106}$ Y. Q. Chen, I. Petráš, and D. Xue, "Fractional order control-A tutorial," in Proceedings of 2009 American Control Conference (IEEE, 2009), pp. 1397-1411.

${ }^{107}$ R. K. H. Galvão, S. Hadjiloucas, K. H. Kienitz, H. M. Paiva, and R. J. M. Afonso, "Fractional order modeling of large three-dimensional RC networks," IEEE Trans. Circuits Syst. I: Regular Pap. 60, 624-637 (2013).

${ }^{108}$ R. K. H. Galvão, J. P. Matsuura, J. R. Colombo, Jr., and S. Hadjiloucas, “Detecting compositional changes in dielectric materials simulated by three-dimensional RC network models," IEEE Trans. Dielectr. Electr. Insul. 24(2), 1141-1152 (2017).

${ }^{109}$ R. K. H. Galvão, K. H. Kienitz, and S. Hadjiloucas, “Conversion of descriptor representations to state-space form: An extension of the shuffle algorithm," Int. J. Control 91(10), 2199-2213 (2017). 